\title{
ESR position paper on ultrasound
}

\author{
ESR Executive Council 2009 • European Society of Radiology
}

Received: 20 October 2009 / Accepted: 18 November 2009 / Published online: 16 January 2010

(C) European Society of Radiology 2009

\begin{abstract}
This paper re-emphasises the important role of diagnostic ultrasound. It underlines the importance of ultrasound as a non-invasive imaging procedure which does not use ionising radiation. The paper also illustrates how ultrasound is often the cornerstone of the work up of the patient and the need for radiology departments to be able to offer expert ultrasound on a $24 / 7$ basis. Some aspects related to turf battles and education are discussed. Finally the way in which these issues will be addressed by the European Society of Radiology is outlined.
\end{abstract}

Keywords Ultrasound · Training · Organisation

\section{General considerations}

Diagnostic ultrasound (US) is a medical imaging technique which, since its introduction in clinical practice at the end of the 1960s, has seen continuous improvement in image quality and constant growth in number of examinations perfromed and variety of clinical applications. It is now considered to be the second most frequently performed imaging examination, following the chest $\mathrm{X}$-ray.

Although radiologists are deeply involved in this field, US is also used extensively by many specialists of other disciplines. It is considered that, nowadays, more than $50 \%$ of US examinations worldwide are performed by clinical specialists rather than radiologists or technicians working in a department of radiology. There are important fields of

ESR Executive Council $2009 \cdot$ European Society of Radiology $(\bowtie)$ Neutorgasse 9/2,

1010 Vienna, Austria

e-mail: communications@myESR.org application of diagnostic US, for instance cardiology, obstetrics, and gynaecology, which are almost exclusively performed by non-radiology specialists.

Turf battles about the use of US continue to grow as more and more specialists are claiming US as part of their everyday's work, and the position of radiologists is progressively further undermined.

It is well know that, in clinical medicine, a strong position in any examination technique belongs to those who are most active in research and new developments. On the radiologists' side, there is a decrease in dedication and involvement in US research. Radiologists probably do most of the "routine" work in US, but seem to pay relatively little attention to the new advances in this field. The most important of them, the use of US contrast media, is growing very slowly and seems more frequently employed by non radiology physicians.

Furthermore, there seems to be lower attention to US also in clinical practice. Young radiologists often regard US as less attractive than CT and MR. Many consider it as a "mature" technique, with few possibilities of advancement and as a physically and mentally stressful activity (high number of requests, continuous "clinical" attention while performing the studies, direct contact with patients). Clinical Directors of Radiology Departments have organization and budget concerns about US: it is an examination technique which is difficult to standardise and consumes considerable human resources (even when performed by a sonographer the physician must be nearby, and "medical time" is costly); CTs and MRs are requested also after US performed by clinicians, and these examinations ensure higher income to the Department. CT and MR often have higher sensitivity and always have wider capabilities: it is commonly thought that the examination technique which, in theory, provides the best results "in one stop" is the best choice. 
In addition, the shift to a practice and academic model in which radiologists are focused on organ systems has, in some ways, highly enhanced the capabilities of radiologists to correlate with referring specialists but, on the other hand, has lowered the visibility of technique based specialities (e.g. US) This has led to decreased emphasis on the technical aspects of US during residency programmes and increased requests for specific ultrasound skills to be obtained towards the end of training.

\section{Advantages of radiological US}

From the patient's perspective, US examinations performed by radiologists carry certain advantages over those performed by other physicians. Radiologists are trained to analyze images on their own merits, not as a means of confirming a diagnosis already supported by clinical findings. Radiologists are better at correlating US results with those of other imaging investigations to reach a final diagnosis. Radiologists are fully dedicated to imaging, which is impossible for a clinician who is involved actively in patient care; thus radiologists are better versed with regards to normal variants and abnormal findings outside the specialist skill of the physician. For example the cardiologist may not be at all interested in the HRCT findings in the chest and may not spot an incidental lung carcinoma.

From the perspective of our discipline, it must be emphasised that, in US practice, there is direct patientphysician interaction. In this era in which there is concern about the "visibility" of the specialty of radiology by the public, direct contact with the patient during the US examinations emphasises that radiology is a clinical discipline, and that it is the radiologist, not the machine, who makes the diagnosis.

Furthermore, US is a non-invasive imaging procedure which does not use ionizing radiation. There is increasing debate about medical irradiation issues in the medical literature and in the lay press; radiologists are strongly advised to use alternative techniques that do not use ionizing radiation whenever possible. In the future, the profession may well be questioned more closely about radiation dose and it would be a pity if we have discarded responsibility for US, which reigns supreme with regard to low radiation risk and safety.

In addition, the future of US looks bright in term of research opportunities and increased clinical applications. Recent technical advances (e.g. Ultrasound perfusion) have significantly boosted the research horizons in this field. As radiologists, we are the best suited specialists to evaluate their technical and clinical interest in comparison with other imaging methods.

\section{Moving into action}

In order to meet all the challenges facing radiological US from both outside and inside radiology, it is necessary to promote increased attention to this technique. We believe this should involve the whole radiological community at professional, educational and Society levels.

\section{Impact on the organization of radiological departments}

In many clinical applications, US is the corner stone of the work-up of the patient. Although a shortage of radiologists can be a serious problem, the organization of the Radiology Department should strive to provide coverage of US services $24 \mathrm{hrs} /$ day. The contribution of sonographers has to be considered in this case.

In the current organization in which radiology is increasingly becoming structured along organ-based lines, there is danger of giving less attention to the technical aspects of our profession. US is a highly operatordependent technique, whose results are critically dependent on practical expertise. To reach and maintain leadership in clinical US, it is therefore necessary that radiology become real masters of the technique and keep it at its forefront. Organ-based radiologists who use this technique extensively, each in their own field, should organize to work closely together on the management and development of the service, ideally with a designated Ultrasound leader. Together, they should be responsible for the quality assessment of the examinations, the purchase of equipment and the training of sonographers and radiologists. Most importantly, they should take care that all the aspects of US, including new developments, are appropriately covered within the department.

As regards budget concerns, the reinbursement for US examinations varies extensively among countries, and for some applications is often insufficient to cover the time and costs of the studies (i.e. contrast-enhanced US). However, an appropriate management of US including, whenever authorized, a contribution of sonographers, may allow the generation of sufficient income to cover expenses. This has to be analyzed. Furthermore, it may be possible to take advantages from new developments too. 3D US, for instance, has been shown capable of shortening examination times, with faster data acquisition and faster throughput of patients.

\section{Dealing with turf battles}

Turf battles between radiologists and clinical specialists affect all aspects of medical imaging in wards and clinics. 
However, they seem especially acute with regards to Ultrasound. Even in hospitals in which radiologists are very active in this technique, US machines are available and used also in many clinical wards. On this point it is always disappointing to see a top-of-the-range ultrasound machine owned by a non-radiologist sitting idly and just used for 2-3 patients per day, while Radiology Departments cannot raise the necessary funds for a new machine which will be used for thousands of patients per annum!

There are two ways to cope with this problem. The first is to strive to keep all US examinations within radiology and to "deny" other specialists the right to use this valuable technique (perhaps on the grounds that they have not been trained in imaging). The second is to work together in order to improve quality of US services within both radiology and clinical specialities. The second way is probably the correct one, but is the most difficult, since it implies technical, organizational and clinical cooperation. Both radiologists and non-radiologists should then cooperate, both within each hospital, and at the level of Scientific Societies, and work on a variety of aspects such as the production of equipment standards, examinations protocols, and reporting and archiving. They should cooperate to organize clinical continuity of services, even for the most sophisticated examinations (there is no point in being able to offer something like endoscopic ultrasound on certain days of the week only). Above all, cooperation should be aimed at the development of both training programs and of guidelines to US imaging. This will ensure high quality standards of examinations and proper access to services. Good guidelines can even influence the self-referral phenomenon, which has been shown to increase health care costs. The present situation sees a high degree of decentralization of US equipment in clinical wards. Working along the same guidelines and with the same operative standards will help provide a comprehensive service.

\section{Impact on education}

Discussion and cooperation has to occur among peers.

Then, radiologists should go to the forefront of this technique and maintain the position.

Young radiologists licensed from certificated residency programmes are well trained in US and are able to perform the most common examinations, but "advanced" US procedures, such as contrast-enhaced studies, musculoskeletal examinations or transvaginal examinations of the female pelvis, are often relatively neglected in radiology, and other imaging techniques such as CT or MR are preferred to evaluate patients who could be effectively imaged by US. If radiologists want to keep a key role in this imaging technique in the future, it cannot be regarded any more as the "poor relation" of CT and MR. The most important aspect should be increased attention to the achievement of full training in all aspects of US during the residency programmes. Even in system-based rotations, training should be arranged in such a way that residents have access to the full range of US applications and "live, eat and breathe" US for long enough to master them. Next, there should be increased attention to US in local clinicoradiological and National/International meetings, with sessions dedicated to advanced US techniques at ECR. Furthermore, specific courses on these topics should be organized for radiologists. These should provide theoretical training on web-based systems, and could be complemented by periods of practical training at centres where such activities are actively performed. As an example, fellowship programmes dedicated to contrast-enhaced US in different organs or systems could be activated to ensure the proper use of this diagnostic technique in all radiology departments.

\section{The European Society of Radiology}

Special attention to radiological US is given by the European Society of Radiology, and the establishment of a short-term working group dedicated to this technique has been created. Its activities shall aim at the coordination of educational programmes on this topic within the radiological community, at setting up examinations standards and protocols to enhance quality of our services and, possibly, at coordinating research programmes in advanced US techniques. To evaluate the role of US, especially its cost effectiveness, in comparison to other imaging techniques is a task that we, as radiologists, are especially well prepared to deal with. Furthermore, the working group should facilitate liason between the ESR and other Scientific Societies, especially those dealing specifically with US, to ensure that the voice of Radiology in this field is clearly heard.

Acknowledgement Paper prepared by the ESR Working Group on Ultrasound.

Members: M. Claudon, L.E. Derchi

Approved by the ESR Executive Council, June 2009. 